Revista de Psicología Vol. 37 (1), 2019 (ISSN 0254-9247)

\title{
Validación del instrumento de compromiso organizacional en México: evidencias de validez de constructo, criterio y confiabilidad
}

\author{
Jessica M. G. Fonseca Herrera ${ }^{1}$, Christian Enrique Cruz Torres ${ }^{2}$, \\ Leticia Chacón Gutiérrez ${ }^{3}$ \\ Instituto Politécnico Nacional, México ${ }^{1}$, Universidad de Guanajuato, México ${ }^{1,2}$, \\ Universidad de la Salle Bajio, México ${ }^{3}$
}

El objetivo del presente estudio fue presentar evidencias de validez de constructo, criterio y consistencia interna de un instrumento que mide el compromiso organizacional. Primero se desarrolló un ejercicio de redes semánticas naturales modificadas para elaborar un banco de reactivos, que posteriormente fueron validados por jueces expertos. En el segundo estudio se probó el instrumento conformado por 40 reactivos con una muestra de 140 trabajadores de una universidad pública del Estado de Guanajuato. Los resultados de este estudio permitieron identificar cuatro factores, observándose niveles adecuados de consistencia interna. Para la tercera fase se aplicó el instrumento final compuesto por 25 reactivos a 230 trabajadores de otra universidad pública, identificándose tres factores (afectivo, moral e identificación con objetivos y valores) con un $\alpha$ de .86 a .89. Los factores encontrados en el trabajo son teóricamente congruentes. Se discuten sus posibles usos, alcances y limitaciones. Palabras clave: compromiso organizacional, medición del compromiso organizacional, validación psicométrica.

1 Licenciada en Psicología. Analista de sistemas y procesos en la Coordinación de Enlace y Gestión Técnica de la Unidad Profesional Interdisciplinaria de Ingenierías del Instituto Politécnico Nacional, Campus Guanajuato. Dirección postal: Instituto Politécnico Nacional, Silao de la Victoria, Guanajuato CP 36750. Contacto: jfonsecah84@gmail.com; jfonsecah@ ipn.mx. ORCID: https://orcid.org/0000-0001-5809-7069

2 Doctor en Psicología. Profesor asociado en la Universidad de Guanajuato. Dirección postal: Blvd. Puente Milenio 1001, Fracción del Predio San Carlos, 37670 León, Gto. Departamento de Psicología, edificio de Ciencias de la Salud. Contacto: cassiel.79@gmail.com ORCID: https://orcid.org/0000-0002-4286-4697

3 Doctora en Pedagogía. Coordinadora del Centro de Neurociencias en la Universidad de la Salle Bajío. Dirección postal: Av. Universidad No. 602 Col. Lomas del Campestre C.P. 37150, León, Guanajuato. Centro de Neurociencias y Escuela de Educación y Desarrollo Humano. Contacto: lchacon@delasalle.edu.mx ORCID: https://orcid.org/0000-0002-7268-200X 
Validation of the Instrument of Organizational Commitment in Mexico: Evidence of construct validity, criterion validity and reliability

The aim of this study was to provide evidence of construct validity, criterion validity, and internal consistency of an instrument that measures organizational commitment. First, an exercise with natural semantic networks was developed to get a pool of items, which were subsequently validated by judges. The second study was a first test of the instrument with 40 items, in a sample of 140 workers of a public university in the state of Guanajuato, Mexico. The results of the second study identify four factors with adequate levels of reliability. For the third study, the final instrument composed of 25 items was answered by 230 workers of a second public university, identifying three factors (affective, moral and identification with goals and values) with reliability indexes of $\alpha$ of .86 to .89 . These factors are theoretically consistent. We discuss possible uses, scope and limitations of the final instrument.

Keywords: organizational commitment, measurement of organizational commitment, psychometric validation.

Validaçáo do instrumento de compromisso organizacional no México: evidência de validade de construçáo, critério e confiabilidade

O objetivo do presente estudo foi apresentar evidências de validade de construçáo, critério e consistência interna de um instrumento que mede o compromisso organizacional. Primeiro, desenvolveu-se um exercício de redes semânticas naturais modificadas para elaborar um banco de reagentes, posteriormente validado por juízes. No segundo estudo, o instrumento foi testado por 40 reagentes com uma amostra de 140 trabalhadores de uma universidade pública do estado de Guanajuato. Os resultados deste estudo permitiram a identificação de quatro fatores, com níveis adequados de consistência interna. Para a terceira fase, o instrumento final foi composto por 25 reagentes para 230 trabalhadores de outra universidade pública, identificando três fatores (afetivos, morais e de identificação com objetivos e valores) com $\alpha=.86$ a .89 . Os fatores encontrados no trabalho são teoricamente congruentes. Seus possíveis usos, escopo e limitaçóes são discutidos.

Palavras-chave: compromisso organizacional, medição de compromisso organizacional, validação psicométrica.

Validation de l'instrument d'engagement organisationnel au Mexique: preuve de la validité conceptuelle, validité du critère et de la fiabilité

Lobjectif de cette étude était de présenter des preuves de la validité conceptuelle, du critère et de la cohérence interne d'un instrument mesurant l'engagement organisationnel. Premièrement, un exercice de réseaux sémantiques naturels modifiés a été mis au point pour préparer une banque de réactifs, qui ont ensuite été validés par des juges experts. Dans la deuxième étude, l'instrument composé de 40 items a été testé avec un échantillon de 140 travailleurs d'une université publique de l'État de Guanajuato. Les résultats de cette étude ont permis d'identifier quatre facteurs, en observant des niveaux adéquats de cohérence interne. Pour la troisième phase, l'instrument final composé de 25 réactifs a été appliqué à 230 travailleurs d'une autre université publique, identifiant trois facteurs (affectif, moral et identification avec des objectifs et des valeurs) avec un $\alpha$ de .86 à .89. Les facteurs trouvés dans le travail sont théoriquement congruents. Ses utilisations possibles, sa portée et ses limites sont discutées. Mots clés: engagement organisationnel, mesure de l'engagement organisationnel, validation psychométrique. 
En las últimas décadas se ha prestado particular interés al estudio del comportamiento de las personas en el campo laboral, al reconocer que los empleados y el ambiente en que trabajan son de vital importancia para el progreso de cualquier organización. De acuerdo con Werther y Davis (2000) "para lograr una mejoría a largo plazo en la productividad es indispensable mejorar la calidad del entorno laboral" (p. 439), es por ello que resulta atractivo para los directivos invertir en actividades que desarrollen el potencial humano y mejoren el ambiente de sus organizaciones. Cada organización requiere ser atendida con base en sus propias características y necesidades. El logro de objetivos, misión y visión están determinados por la relación que se establece entre sus integrantes, así como por la forma en que se utilizan los recursos con que cuentan y el trabajo realizado (Davis, 1983). A fin de llevar acabo las funciones que como grupo social establezcan, las organizaciones dependen de la sistematización de sus recursos en relación con la disposición, correlación y jerarquías instauradas (Münch \& García, 1990). Otros aspectos del funcionamiento de una organización a considerar son la comunicación entre sus miembros, las normas de conducta que las rigen y la disposición de las personas para actuar de manera coordinada a fin de lograr la meta establecida. En general, el rumbo de cualquier organización está influenciado por el ambiente de trabajo en el que se encuentran inmersas las personas.

La percepción que tienen los empleados acerca de la organización en que laboran hace referencia al ambiente de trabajo (Robbins, 1996), el cual también es conocido como clima organizacional. Para Davis (1983) es definido como "el ambiente humano dentro del cual realizan su trabajo los empleados e influye en la motivación, el desempeño y la satisfacción en el empleo" (p. 111). El clima o ambiente de trabajo es creado por las personas que conforman la organización con sus acciones y percepciones; se genera a partir de sus expectativas sobre 
recompensas, satisfacciones, reconocimiento, incluso frustraciones o consecuencias negativas por su comportamiento en la organización.

El ambiente en la organización es dinámico, cambia y se ve influido por las características y las acciones de los empleados. De acuerdo con Davis (1983) para crear un clima favorable existen elementos clave a tomar en cuenta, entre los que destacan el liderazgo, los sentimientos de los empleados acerca de realizar un trabajo útil, la responsabilidad compartida con la organización, las oportunidades de crecimiento y el compromiso de los integrantes. Este último es denominado compromiso organizacional $(\mathrm{CO})$ y resulta fundamental para el crecimiento de las organizaciones (Gómez-Sánchez, Recio \& Avalos, 2012) y por lo tanto ha sido constantemente estudiado.

En el marco del estudio del $\mathrm{CO}$ se han identificado múltiples definiciones que lo consideran como una actitud que deriva en un comportamiento hacia la organización. A continuación se mencionan algunas:

Según Mowday, Steers y Porter (1979) el CO es "la fuerza relativa a la identificación de un individuo con, e implicación en, una organización particular" (p. 226), y se caracteriza por tres factores de relación: (a) aceptación y creencia en los valores y objetivos institucionales, (b) la voluntad de las personas para esforzarse en nombre de su institución y (c) el deseo de mantener su membresía en la organización. Por su parte Meyer y Allen (1991) lo definen como un "estado psicológico que caracteriza la relación entre una persona y una organización” (p. 62), reflejando una necesidad, un deseo y/o una obligación de los empleados a fin de mantener su pertenencia con la organización. De acuerdo con Robbins (1996) el CO se refiere al grado en que un empleado se verá identificado con la organización a la que pertenece, buscando además sostener dicha pertenencia.

Las diferentes definiciones del $\mathrm{CO}$ mostradas exponen un componente actitudinal que los empleados presentan hacia la organización a la que pertenecen, respondiendo así a las distintas fuentes dentro de la literatura. Aunque se pueden identificar diferencias entre las definiciones presentadas, los estudios en general muestran al compromiso 
organizacional como un elemento positivo para la relación entre los empleados y su organización. Por ejemplo Gómez-Sánchez, Recio y Avalos (2012) mencionan que el compromiso organizacional está en estrecha relación con la satisfacción con el trabajo y la productividad de las personas. Por otra parte, se ha encontrado que cuando las personas no se comprometen con su organización tienden a presentar más ausentismos (Lu, While \& Barriball, 2005).

La estructura del constructo así como las definiciones han ido variando con el paso del tiempo; de acuerdo a las explicaciones y estudios realizados por Mowday, Steers y Porter (1979), se consideraba que el $\mathrm{CO}$ era unidimensional. En la actualidad existe una corriente que considera al $\mathrm{CO}$ como un constructo multidimensional, sin embargo no existe un acuerdo generalizado en cuanto a la cantidad de dimensiones o factores (Betanzos, Andrade \& Paz, 2006; Frutos, Ruiz \& San Martín, 1998).

Distintos tipos de compromiso interactúan entre sí, por lo que una persona es capaz de presentar al mismo tiempo y con la misma intensidad las diferentes formas del compromiso (Barraza \& Acosta, 2008; Frutos et al., 1998). Algunos autores plantean la existencia de cinco formas del compromiso en el trabajo (Cohen, 1999; McElroy, Morrow \& Laczniak, 2001; Morrow, 1983; Morrow \& McElroy, 1986). Los cuales presentan distintos enfoques entre sí: (a) en el valor, (b) en la carrera, (c) en el trabajo, (d) organizacional y (e) sindical. Bajo esta perspectiva se identifican componentes relacionados con la vivencia diaria en los ambientes de trabajo y a la vez cuestiones dadas por la cultura y la profesión.

Otros estudios refieren una estructura del CO integrada por tres componentes o dimensiones, siendo: (a) compromiso afectivo, el cual se refiere a la atracción emocional, a la identificación y al involucramiento del empleado con la organización, es decir el empleado permanece en la organización porque desea hacerlo; (b) el compromiso de continuidad o por conveniencia, se refiere a la conciencia de la persona respecto a la inversión en tiempo y esfuerzo que se perderían en caso de dejar la organización para encontrar otro empleo, es decir 
permanece en la organización porque lo necesita; y (c) el compromiso normativo, el cual refleja un sentimiento de obligación para permanecer, luego entonces de quedarse en ella (Allen \& Meyer, 1990, 1996; Barraza \& Acosta, 2008; Frutos et al., 1998; Meyer \& Allen, 1991; Meyer \& Herscovitch, 2001).

Se han desarrollado instrumentos para medir el CO dando respuesta a las diferentes visiones y de acuerdo a la concepción de la época. En 1974, Porter y sus colaboradores (citado en Betanzos et al., 2006) elaboraron el Cuestionario de compromiso organizacional que identifica un solo factor o dimensión. Luego de un par de décadas, Allen y Meyer (1990) crearon otro instrumento, donde consideran tres factores que componen el CO: (a) afectivo $(\alpha=.87)$, (b) continuidad $(\alpha=.75)$ y (c) normativo $(\alpha=.79)$, el cual permanece vigente en su uso y con amplio reconocimiento.

En América Latina se han desarrollado adaptaciones de la validación realizada por Frutos et al. (1998), manteniendo los tres componentes: afectivo, normativo y de continuidad. En México se han reportado distintos índices de consistencia interna en los mismos factores, en la versión con 18 ítems validada en 1998 por Arias (citado en Arias, 2001) se obtuvieron valores de $\alpha=.71$ a .81 para el componente afectivo, de $\alpha=.47$ a .48 para el componente de continuidad y de $\alpha=.63$ a .82 para el componente normativo. Por otra parte, Barraza y Acosta (2008) reportaron 21 reactivos y una consistencia interna de .74 en el componente afectivo, de .79 para el componente de continuidad y de .67 para el componente normativo.

Al encontrar en la literatura tantas definiciones, estructuras teóricas y variaciones en la cantidad de reactivos utilizados, y considerando que en su mayoría reflejan el trabajo realizado en contextos distintos al mexicano, surge la necesidad de contar con un instrumento que mida el CO para la población mexicana y que por ende cuente con validez cultural. El objetivo de este estudio fue presentar evidencias de validez de constructo, criterio y consistencia interna de un instrumento diseńado para medir el $\mathrm{CO}$ en una muestra de trabajadores mexicanos. Con la intención de obtener validez de constructo, criterio y 
consistencia interna del instrumento que mide el CO en México se realizaron tres estudios. El primero fue exploratorio, se utilizó la técnica de redes semánticas naturales modificadas para obtener reactivos culturalmente relevantes (Reyes, 1993; Vera, Pimentel \& Batista, 2005); en el segundo a fin de obtener evidencias de validez de constructo, se utilizó un análisis factorial exploratorio; y el tercero tuvo como objetivo fortalecer la validez de constructo, criterio y consistencia interna mediante un análisis factorial confirmatorio mediante ecuaciones estructurales y un análisis de comparación multigrupos para poner a prueba la equivalencia del instrumento en las dos submuestras estudiadas.

\section{Método}

\section{Participantes}

En el estudio número uno, se contó con una participación de 40 empleados de una universidad pública que es referente de la educación a nivel superior en México, recientemente insertada en el Estado de Guanajuato. De manera intencional los participantes fueron $50 \%$ hombres y $50 \%$ mujeres, asimismo la distribución referente a la actividad que desarrollan fue $50 \%$ personal docente y el resto fueron administrativos.

Para el segundo participaron 140 empleados de la plantilla de otra universidad pública del Estado arraigada y con prestigio en la región, de los cuales 79 fueron hombres y 61 mujeres; $74 \%$ fueron docentes y el 26\% restante personal administrativo; el 58\% de la muestra contaba con una antigüedad entre uno y cinco años, el $43 \%$ restante con más de seis ańos trabajando en la universidad.

En el tercer estudio se contó con la participación de 230 empleados de la misma universidad pública, aunque 180 de ellos laboran en la sede recientemente insertada en el estado de Guanajuato y 50 laboran en la sede de la Ciudad de México. La distribución de los participantes fue $49 \%$ hombres y $51 \%$ mujeres; 133 fueron personal docente y 90 personal administrativo; el $6.3 \%$ cuenta con menos de un ańo de antigüedad, el 59.2\% tienen entre uno y cinco ańos en la universidad, 
el $18.4 \%$ reportaron tener entre seis y 10 ańos de antigüedad y el $16.1 \%$ restante tienen más de 11 años en la universidad.

\section{Medición}

En el primer estudio se aplicó la técnica de redes semánticas naturales modificadas, la cual tiene como objetivo identificar el significado psicológico referente a algún constructo para una población determinada con base en la reiteración de palabras por parte de los participantes (Reyes, 1993; Vera, Pimentel \& Batista, 2005). Los estímulos escritos utilizados para la técnica fueron: (a) Compromiso organizacional es, (b) Para mi, ser (parte de la institución X) significa y (c) las normas institucionales son.

Para el segundo estudio se utilizó el instrumento que se construyó a partir de los resultados de redes semánticas naturales modificadas, compuesto por 40 reactivos medidos en una escala tipo Likert pictórico con cinco opciones de respuesta que van de totalmente de acuerdo a totalmente en desacuerdo, que integran cuatro componentes del CO: (a) afectivo, referente al disfrute que presentan los empleados por pertenecer en la organización (e.g. Disfruto mucho trabajar en esta organización; Trabajar aqui es muy importante para mi). (b) moral, definido como la lealtad del empleado hacia la organización (e.g. Esta institución merece mi lealtad; Me sentiría culpable si dejara esta institución). (c) recompensa directa, sobre un análisis del costo beneficio resultado de estar en la organización en comparación con la posibilidad de estar en otra (e.g. En esta institución tengo mayores beneficios que en otras; Esta institución me brinda todo lo que necesito). Y (d) identificación con objetivos y valores, en la medida en que el empleado internaliza los valores y objetivos de la organización y se siente identificado con estos (e.g. Mi trabajo aporta significativamente a los objetivos de esta institución; Me identifico con los objetivos de esta institución).

Para el tercer estudio se utilizó el instrumento compuesto por 25 reactivos, 14 resultantes de la estructura factorial del segundo estudio y once reactivos que se incorporaron para fortalecer la estructura 
teóricamente esperada de las cuatro dimensiones del instrumento, considerando que por tratarse de instituciones diferentes podrían tener estructuras factoriales distintas.

Con la intención de obtener evidencias de validez de criterio, se aplicó además el instrumento BIARE (instrumento desarrollado por el INEGI en 2012 para medir de manera operativa el bienestar subjetivo) y que entre sus componentes aportan información sobre la satisfacción en el trabajo de las personas en una escala de 0 a 10.

\section{Procedimiento}

En los tres eventos la participación de los empleados de las universidades fue voluntaria, se les informó la finalidad del estudio correspondiente y se hizo hincapié en el tratamiento confidencial de los resultados. La mayoría de las aplicaciones se realizaron de forma individual, sin embargo también se realizaron aplicaciones grupales de acuerdo con la disponibilidad del personal y sus cargas de trabajo. Se obtuvieron las autorizaciones pertinentes con los directivos de cada universidad de forma escrita para realizar los estudios, evitando el uso de los medios internos de comunicación y de los logos institucionales de acuerdo a lo solicitado por las autoridades.

\section{Análisis de datos}

La técnica de redes semánticas naturales modificadas utilizada en el primer estudio permite identificar el sentido o significado que las personas le dan a cada estímulo presentado. Dicha técnica ha sido empleada en distintos fenómenos cognoscitivos en México (Figueroa, González \& Solís, 1981; Hinojosa, 2008; Reyes, 1993; Vera, Pimentel $\&$ Batista, 2005). Para la aplicación de la técnica a los participantes se les presenta una serie de estímulos escritos (e.g. oraciones, términos específicos) de las cuales producen una lista de al menos cinco palabras definidoras (palabras que describen el estímulo presentado), a cada una de estas les asignan un peso (valor semántico), con base en la importancia jerárquica como definidora del estímulo para el participante. 
El cálculo de resultados de la técnica se estima haciendo una relación de todas las palabras definidoras dadas por los participantes, al total de palabras se le considera valor $J$. Otro valor generado es el peso semántico de cada palabra definidora (valor $M$ ), el cual se obtiene de multiplicar la frecuencia de aparición en cada lugar de la jerarquía por el valor semántico obtenido. Las palabras encontradas en primer lugar son las que más peso tendrán. Después se grafica la frecuencia de aparición y peso semántico de las palabras a fin de identificar el núcleo de la red, el cual se establece al identificar visualmente en la gráfica el punto en el cual línea se vuelve asintótica al eje horizontal (Figueroa, González \& Solís, 1981; Hinojosa, 2008; Reyes, 1993).

\section{Resultados}

Como resultado de la técnica de redes semánticas naturales modificadas, con respecto a la población participante se obtuvo para el estímulo Compromiso organizacional es, un tamaño de red de 102 palabras definidoras con un núcleo de red formado por siete palabras (responsabilidad, respeto, trabajo, compromiso, comunicación, honestidad y orden). Con el segundo estímulo "Para mí, ser __ significa", se obtuvo un tamaño de red de 93 definidoras y un núcleo de siete palabras (compromiso, orgullo, trabajo, servicio, identidad, responsabilidad y lealtad). Finalmente el tercer estímulo "Las normas institucionales son", presentó un tamaño de red de 118 definidoras y un núcleo mayor que los estímulos previos, las palabras que lo integraron son: reglas, compromiso, respeto, orden, necesarias, regulación, lineamientos, honestidad.

Las palabras definidoras asociadas al significado psicológico del constructo en general fueron: compromiso, comunicación, honestidad, identidad, lealtad, lineamientos, orden, orgullo, reglas, regulación, respeto, responsabilidad, servicio y trabajo. Con base en estos resultados y las definiciones teóricas se elaboró un banco de reactivos diseñados para medir el CO. Se ajustó la redacción de los reactivos buscando mayor 
claridad y se sometieron a análisis para validez de contenido mediante la técnica de jueceo, obteniéndose 40 reactivos diseñados para medir las cuatro dimensiones del CO.

En el segundo estudio se puso a prueba la consistencia interna y validez de constructo del instrumento. Iniciando con un análisis de frecuencias de los reactivos, a fin de conocer el grado de discriminación individual de los mismos (Reyes, 1993). Posteriormente los reactivos que obtuvieron buenos indicadores de discriminación fueron expuestos a un análisis factorial exploratorio a través del método de máxima verosimilitud con rotación ortogonal varimax a fin de poner a prueba la validez de constructo. Los reactivos se agruparon en los cuatro factores propuestos inicialmente: afectivo, recompensa directa, moral, e identificación con objetivos y valores. Se obtuvo una varianza total explicada de 63.64\%, con un indicador de bondad de ajuste $X^{2}=76.625, \mathrm{gl}=41$, $p=.001$.

El siguiente paso fue realizar una prueba de consistencia interna para cada factor mediante la fórmula alfa de Cronbach, obteniéndose valores que se mantienen dentro de rangos adecuados (>.80) (Tabla 1).

\section{Tabla 1}

Análisis factorial exploratorio de instrumento compromiso organizacional. Estudio 2

\begin{tabular}{lcccc}
\hline & Afectivo & Moral & $\begin{array}{r}\text { Identificación con } \\
\text { objetivos y valores }\end{array}$ & $\begin{array}{c}\text { Recompensa } \\
\text { directa }\end{array}$ \\
\hline$M$ & 4.310 & 2.666 & 4.206 & 3.508 \\
$D E$ & .610 & 1.461 & .675 & 1.290 \\
Varianza explicada & $17.25 \%$ & $16.24 \%$ & $15.87 \%$ & $13.99 \%$ \\
$\alpha$ & .839 & .811 & .863 & .792 \\
\hline Reactivos & & & & .218 \\
\hline $\begin{array}{l}\text { Me gusta estar en esta } \\
\text { institución }\end{array}$ & $\mathbf{. 8 8 3}$ & & .094 & .116 \\
$\begin{array}{l}\text { Disfruto mucho trabajar } \\
\text { en esta institución }\end{array}$ & $\mathbf{. 7 5 5}$ & & &
\end{tabular}




\begin{tabular}{|c|c|c|c|c|}
\hline & Afectivo & Moral & $\begin{array}{l}\text { Identificación con } \\
\text { objetivos y valores }\end{array}$ & $\begin{array}{c}\text { Recompensa } \\
\text { directa }\end{array}$ \\
\hline $\begin{array}{l}\text { Trabajar en esta institución } \\
\text { me hace sentir bien porque } \\
\text { me permite desarrollarme } \\
\text { personalmente }\end{array}$ & .664 & & .199 & .371 \\
\hline $\begin{array}{l}\text { Trabajar aquí es muy } \\
\text { importante para mí }\end{array}$ & .520 & .208 & .362 & .090 \\
\hline $\begin{array}{l}\text { Me sentiría culpable si } \\
\text { dejara esta institución }\end{array}$ & & .838 & .110 & .094 \\
\hline $\begin{array}{l}\text { Aunque me ofrecieran otro } \\
\text { empleo, no sería bueno } \\
\text { irme de esta institución }\end{array}$ & .104 & .733 & .109 & .092 \\
\hline $\begin{array}{l}\text { Si dejara esta institución } \\
\text { ahora, tendría un gran } \\
\text { costo para mí }\end{array}$ & -.061 & .666 & .097 & .055 \\
\hline $\begin{array}{l}\text { No estaría bien dejar esta } \\
\text { institución porque me ha } \\
\text { dado mucho }\end{array}$ & .144 & .635 & .050 & .353 \\
\hline $\begin{array}{l}\text { Comparto los valores de } \\
\text { esta institución }\end{array}$ & .100 & .214 & .930 & .060 \\
\hline $\begin{array}{l}\text { Me identifico con los } \\
\text { valores de esta institución }\end{array}$ & .381 & .106 & .719 & .222 \\
\hline $\begin{array}{l}\text { Me identifico } \\
\text { con los objetivos de } \\
\text { esta institución }\end{array}$ & .303 & .077 & .719 & .286 \\
\hline $\begin{array}{l}\text { En esta institución valoran } \\
\text { mi trabajo }\end{array}$ & .245 & .144 & .106 & .767 \\
\hline $\begin{array}{l}\text { La inversión de tiempo y } \\
\text { esfuerzo a esta institución } \\
\text { me ha sido recompensada }\end{array}$ & .119 & .089 & .188 & .669 \\
\hline $\begin{array}{l}\text { Esta institución me brinda } \\
\text { todo lo que necesito }\end{array}$ & .189 & .226 & .109 & .662 \\
\hline
\end{tabular}

Nota: Factor de ponderación >.40 en negritas. Método de extracción: Máxima verosimilitud. Método de rotación: Normalización Varimax con Kaiser. La rotación ha convergido en 6 iteraciones. $\alpha$ total $=.857$. 
En el tercer estudio se realizó un análisis factorial exploratorio mediante el método máxima verosimilitud con rotación ortogonal varimax, el cual arrojó tres factores de los cuatro propuestos anteriormente: afectivo, identificación con objetivos y valores y el componente moral. La varianza total explicada en conjunto fue de $57.19 \%$, mostrando un adecuado indicador de bondad de ajuste $\left(^{2}=196.525\right.$, $g l=102, p=.000)$. También se puso a prueba la consistencia interna mediante la fórmula alfa de Cronbach (Tabla 2).

\section{Tabla 2}

Análisis factorial exploratorio de instrumento compromiso organizacional. Estudio 3

\begin{tabular}{|c|c|c|c|}
\hline & Afectivo & $\begin{array}{c}\text { Identificación } \\
\text { con objetivos } \\
\text { y valores }\end{array}$ & Moral \\
\hline$M$ & 3.851 & 4.202 & 3.027 \\
\hline$D E$ & 1.110 & 0.624 & 1.261 \\
\hline Varianza explicada & $21.76 \%$ & $18.50 \%$ & $16.92 \%$ \\
\hline$\alpha$ & .896 & .871 & .862 \\
\hline \multicolumn{4}{|l|}{ Reactivos } \\
\hline $\begin{array}{l}\text { Trabajar en esta institución me hace sentir bien } \\
\text { porque me permite desarrollarme personalmente }\end{array}$ & .784 & .172 & .270 \\
\hline $\begin{array}{l}\text { La inversión de tiempo y esfuerzo a esta } \\
\text { institución me ha sido recompensada }\end{array}$ & .697 & .166 & .298 \\
\hline $\begin{array}{l}\text { Trabajar en esta institución ha valido el esfuerzo } \\
\text { y tiempo invertido }\end{array}$ & .681 & .264 & .217 \\
\hline $\begin{array}{l}\text { Trabajar en esta institución permite que satisfaga } \\
\text { mis necesidades básicas }\end{array}$ & .664 & .193 & .183 \\
\hline Esta institución me brinda todo lo que necesito & .599 & .198 & .371 \\
\hline En esta institución valoran mi trabajo & .597 & .182 & .344 \\
\hline Disfruto mucho trabajar en esta institución & .534 & .399 & .224 \\
\hline Si esta organización crece, crezco yo también & .505 & .285 & .341 \\
\hline Comparto los valores de esta institución & .123 & .870 & .153 \\
\hline
\end{tabular}




\begin{tabular}{|c|c|c|c|}
\hline & Afectivo & $\begin{array}{c}\text { Identificación } \\
\text { con objetivos } \\
\text { y valores }\end{array}$ & Moral \\
\hline Me identifico con los valores de esta institución & .236 & .820 & .121 \\
\hline $\begin{array}{l}\text { Me identifico con los objetivos de esta } \\
\text { institución }\end{array}$ & 247 & .770 & .162 \\
\hline $\begin{array}{l}\text { Trabajo en esta institución porque sus valores } \\
\text { son compatibles con mis valores }\end{array}$ & .154 & .638 & .214 \\
\hline $\begin{array}{l}\text { Respeto las normas de esta institución porque } \\
\text { las considero adecuadas }\end{array}$ & .314 & .496 & .101 \\
\hline Le debo mucho a esta institución & .338 & .286 & .730 \\
\hline Me siento en deuda con esta institución & 218 & .219 & .692 \\
\hline $\begin{array}{l}\text { No estaría bien dejar esta institución porque me } \\
\text { ha dado mucho }\end{array}$ & .328 & .168 & .686 \\
\hline $\begin{array}{l}\text { Lo correcto es permanecer en esta institución } \\
\text { para retribuirle por lo que me ha brindado }\end{array}$ & .330 & .147 & .677 \\
\hline Me sentiría culpable si dejara esta institución & 161 & & .565 \\
\hline
\end{tabular}

Nota: Factor de ponderación >.40 en negritas. Método de extracción: Máxima verosimilitud. La rotación ha convergido en 5 iteraciones. Método de rotación: Normalización Varimax con Kaiser. $\alpha$ total $=.925$

Con la intención de obtener evidencias adicionales de validez de constructo se realizó un análisis factorial confirmatorio mediante ecuaciones estructurales utilizando el programa IBM SPSS AMOS 22 (Arbuckle, 2013). De los resultados del análisis, que se muestran en la Figura 1, es posible determinar que las discrepancias entre el modelo propuesto y el resultante no son estadísticamente significativas $\left(X^{2}=63.516 ; \mathrm{gl}=51 ; p=.112\right)$, por lo que es posible decir que el modelo teórico encuentra respaldo en la distribución de los datos. El indicador RMR tuvo un valor igual a .037 , indicando que existe poca varianza restante después de la explicada por el modelo teórico. El valor para la RMSEA fue de .034, con intervalos de .000 a .058. El CFI fue igual a .989. Estos indicadores en su conjunto dan cuenta de un adecuado ajuste de los datos al modelo teórico de medición (Brown, 2006). 


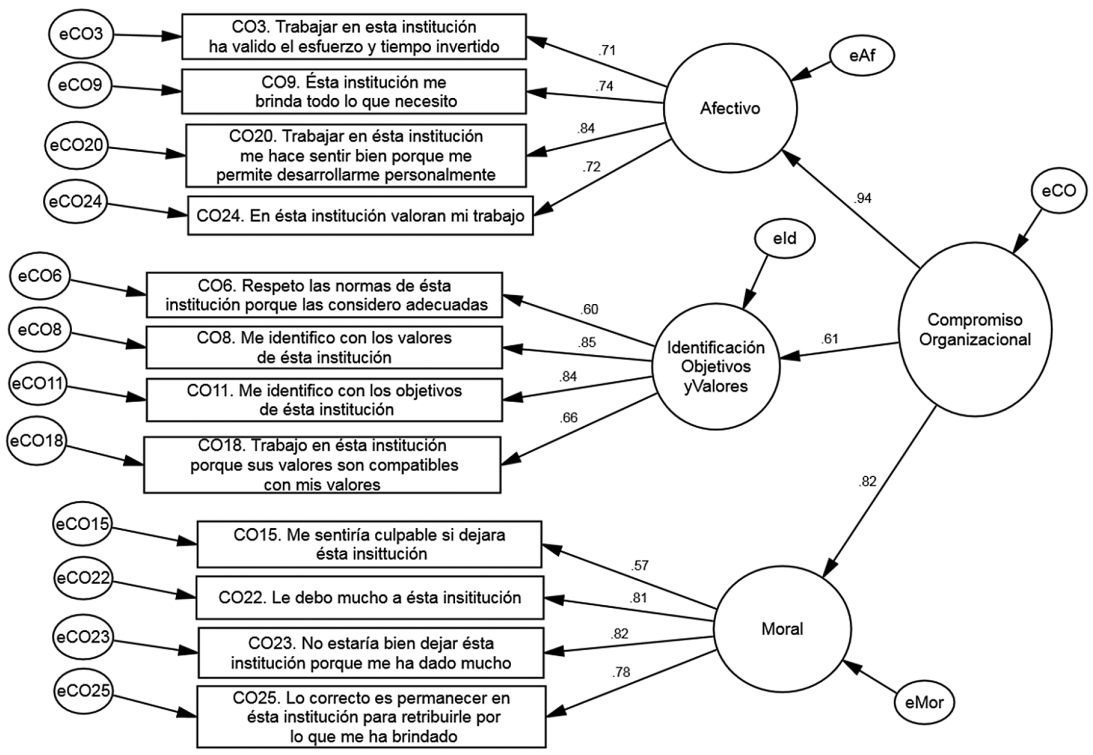

Figura 1. Análisis Factorial Confirmatorio Compromiso Organizacional en Escuela 1.

Este análisis factorial confirmatorio muestra una estructura con tres factores cada uno con cuatro reactivos que en su conjunto permiten medir el CO. El primer factor, afectivo, se refiere a los lazos emocionales que las personas forjan con la organización. Refleja el apego emocional al percibir cubiertas sus expectativas. Los empleados reportan disfrutar de su pertenencia a la organización.

El segundo, identificación con objetivos y valores, es definido como el apego de la persona hacia las normas de la organización. En la medida en que el sujeto internaliza los valores y objetivos de la organización y se siente identificado con estos, buscará el desarrollo tanto de forma personal como el de la organización misma. 


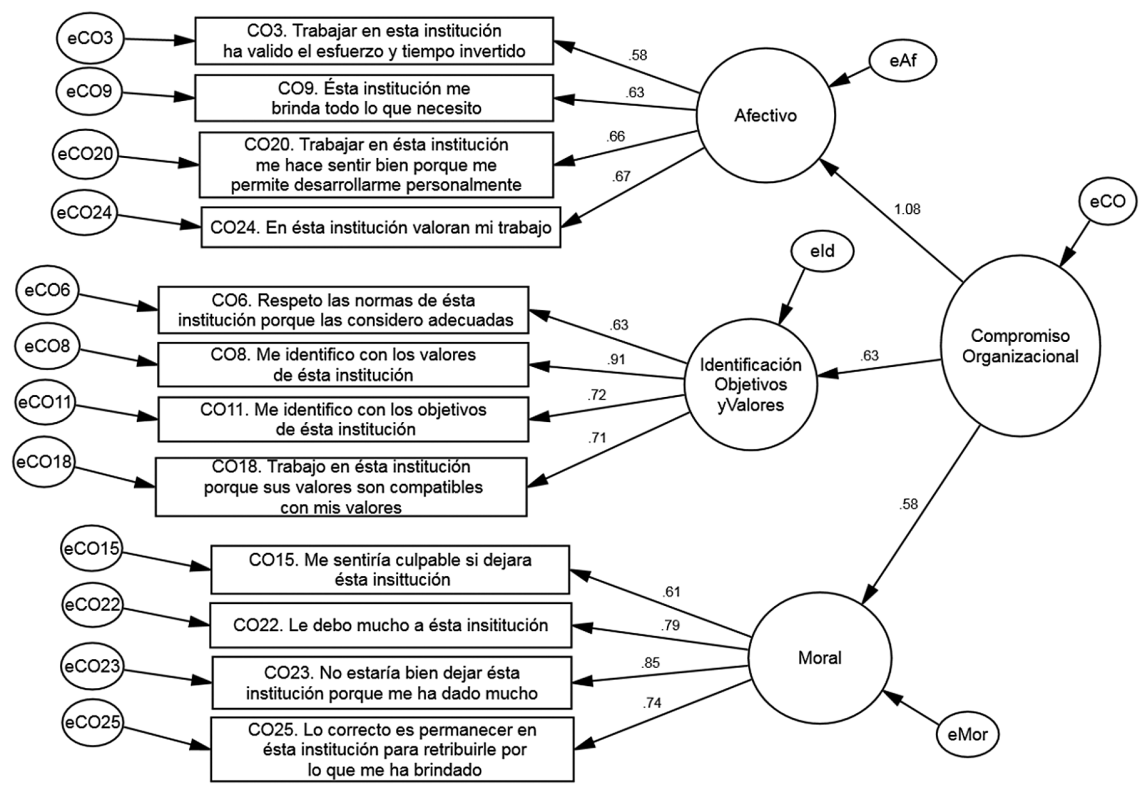

Figura 2. Análisis Factorial Confirmatorio Compromiso Organizacional en Escuela 2.

Nota: los coeficientes de las vías corresponden a coeficientes estandarizados de regresión (betas).

El tercer factor, moral, se define como la lealtad hacia la organización, en un sentido moral, a la existencia de un fuerte vínculo de compromiso con la organización como efecto de una percepción de deuda por la oportunidad recibida; como un pago moral como resultado de percibir que el trabajo es valorado.

Con la intención de poner a prueba la equivalencia de las propiedades psicométricas del instrumento en las submuestras de la sede Guanajuato y la sede Ciudad de México, se realizaron comparaciones multigrupos, también mediante el software IBM SPSS AMOS 22 (Arbuckle, 2013), y se compararon los valores alfa de Cronbach para cada factor mediante el sistema COCRON (Diedenhofen \& Musch, 2016) utilizando la prueba de Feldt y considerando un nivel de confianza del 95\%. Como se observa 
en la tabla 3, los niveles de consistencia interna pueden considerarse equivalentes entre ambas muestras para los factores Moral e Identificación con objetivos y valores, con valores de $\mathrm{Ji}^{2}$ pequeños y no significativos. Por otro lado, el factor afectivo si muestra un funcionamiento diferencial, donde los niveles de consistencia interna son significativamente superiores para la escuela 1 , aunque en ambos casos se encuentran por arriba de un margen aceptable para el valor de alfa de .7.

\section{Tabla 3}

Comparación de valores alpha de Cronbach para cada factor en las muestras de las dos universidades

\begin{tabular}{lccccc}
\hline & Escuela 1 & Escuela 2 & $X^{2}$ & gl & $p$ \\
\hline Afectivo & .832 & .715 & 7.09 & 1 & .007 \\
$\begin{array}{l}\text { Identificación con objetivos y } \\
\text { valores }\end{array}$ & .823 & .824 & .0008 & 1 & .977 \\
Moral & .833 & .833 & .000 & 1 & 1 \\
\hline
\end{tabular}

Comparación realizada con la prueba de Feldt, considerando un nivel de confianza 95\%.

Para la comparación multigrupos se utilizó el software AMOS 22 (Arbuckle, 2013) a fin de poner a prueba la equivalencia del instrumento previamente verificada en el factorial confirmatorio en las muestras de ambas universidades. Al comparar los índices de ajuste del modelo sin restricciones $\left(X^{2}=170.283, \mathrm{gl}=102, p=.000\right)$ y el modelo con restricciones de igualdad en las cargas de medición $\left(X^{2}=189.985\right.$, $\mathrm{gl}=111, p=.000)$ se observan incrementos significativos en los valores de $X^{2}\left(\Delta X^{2}=19.701, \mathrm{gl}=9, p=.020\right)$. Sin embargo, la comparación del CFI entre el modelo sin restricciones de igualdad (CFI=.963) y el modelo con restricciones de igualdad en las cargas de medición $(\mathrm{CFI}=.957)$ permite ver un decremento de .006, menor al .01 sugerido por Cheung y Rensvold (2002) como un valor máximo que permitiría garantizar la equivalencia entre ambos modelos. Al comparar el modelo de medición con el modelo que impone restricciones de igualdad en los interceptos $\left(X^{2}=194.684, \mathrm{gl}=113, p=.000\right)$ se obtienen valores de $X^{2}$ que no 
reflejan diferencias estadísticamente significativas entre ambos modelos $\left(X^{2}=4.699, \mathrm{gl}=2, p=.095\right)$. Al comparar el modelo con restricciones de igualdad en las cargas de medición y el modelo con restricciones de igualdad en los interceptos $(\mathrm{CFI}=.955)$ permite ver un decremento de .008 , nuevamente menor al .01 sugerido por Cheung y Rensvold (2002).

Al comparar el modelo de igualdad en los interceptos con el modelo que impone restricciones de igualdad en los pesos estructurales $\left(X X^{2}=216.455, \mathrm{gl}=117, p=.000\right)$ se obtienen valores de $X^{2}$ que reflejan diferencias estadísticamente significativas entre ambos modelos $\left(X^{2}=21.771, \mathrm{gl}=4, p=.000\right)$. Este resultado coincide con las comparaciones en el CFI, que muestran un decremento de .017 entre el modelo con restricciones de igualdad en los interceptos y el modelo con restricciones de igualdad en los pesos estructurales $(\mathrm{CFI}=.946)$. Los resultados del RMSEA indican un adecuado ajuste incluso para el modelo con restricciones de igualdad en los residuales (RMSEA $=.049$ con rangos mínimo=.035 y máximo=.056, pero dado que los otros indicadores garantizan la equivalencia solo para el nivel de igualdad interceptos, se decidió conservar la comparación hasta ese nivel (RMSEA $=.046$ con rangos mínimo=.035 y máximo=.056), garantizándose únicamente la equivalencia configural y métrica del instrumento.

Para obtener una evidencia adicional de validez de constructo se analizaron las correlaciones entre los tres factores, mediante correlaciones producto momento de Pearson. En congruencia con lo esperado teóricamente, las correlaciones entre los factores son positivas, altas y estadísticamente significativas. Esta relación es especialmente alta entre el factor afectivo y el de identificación con objetivos y valores (Tabla 4).

Con intención de obtener evidencias de validez de criterio se analizaron las correlaciones entre cada factor del $\mathrm{CO}$ con la medición de satisfacción en el trabajo. Los resultados muestran relaciones positivas, altas y estadísticamente significativas para los tres componentes del $\mathrm{CO}$ (afectivo, $r=.674, p<.001$; moral, $r=.497, p<.001$; identificación con objetivos y valores, $r=.465, p<.001$ ), lo cual es congruente con investigaciones previas y la concepción teórica del CO (Mañas, Salvador, Boada, González \& Agulló, 2007). 


\section{Tabla 4}

Correlaciones entre los factores de $\mathrm{CO}$

\begin{tabular}{lccc}
\hline & Afectivo & Moral & $\begin{array}{l}\text { Identificación con } \\
\text { objetivos y valores }\end{array}$ \\
\hline $\begin{array}{l}\text { Afectivo } \\
\text { Moral }\end{array}$ & $-52^{* *}$ & - & \\
$\begin{array}{l}\text { Identificación con } \\
\text { objetivos y valores }\end{array}$ & $.64^{* *}$ & $.43^{* *}$ & \\
\hline
\end{tabular}

Nota: $\mathrm{n}=222$; ${ }^{* *}$ La correlación es significativa en el nivel .01 (bilateral).

\section{Discusión}

La técnica de redes semánticas utilizada en el primer estudio ofreció un insumo de información de primera mano respecto de cómo conciben los participantes el $\mathrm{CO}$ en sus propias organizaciones en México. Esta aproximación permite garantizar que el instrumento aquí presentado cubre el criterio de validez y relevancia cultural (Reyes, 1993; Vera, Pimentel \& Batista, 2005). Esta es una característica importante considerando que la mayoría de los instrumentos encontrados y utilizados en otras investigaciones en México han sido adaptaciones o traducciones elaboradas originalmente en otros países

Coincidiendo con los hallazgos de Betanzos, Andrade y Paz (2006), la aplicación y análisis de resultados del segundo estudio dieron como resultado cuatro factores del CO (afectivo, moral, recompensa directa e identificación con objetivos y valores), es decir, para la población en cuestión tener la camiseta puesta implica involucrarse con la organización, sus normas o lineamientos y pretender un crecimiento mutuo, es decir, con recompensas también para el empleado.

Con el tercer estudio, además de mostrar evidencia de que el CO es un constructo multidimensional, se coincidió en cantidad con los factores planteados por Allen y Meyer (1990), sin embargo se reflejan solo dos de los propuestos por dicho autores ańadiéndose el factor de identificación con objetivos y valores. 
En este estudio como en otros anteriores el componente afectivo del compromiso organizacional aparece de manera constante (Allen \& Meyer, 1990; Blau, 2003; Frutos et al., 1998; Meyer, Stanley, Herscovitch \& Topolnytsky, 2002). El deseo de los empleados de permanecer en la institución en que laboran y la satisfacción que les genera estar ahí son elementos principales del componente afectivo del CO.

Permanecer en una organización por ser leal a la misma refleja el componente moral, que ha sido denominado por otros autores como normativo ya que hace referencia al apego que tiene el empleado a las normas que rigen su comportamiento en la organización en cuestión (Allen \& Meyer, 1996; Betanzos, Andrade \& Paz, 2006; Meyer \& Allen, 1991).

La identificación con los objetivos y los valores institucionales aporta significativamente a la permanencia de los empleados y al compromiso que generan con la organización. En la medida en que los empleados cuenten con valores y objetivos personales afines a su organización buscarán conseguir lo planeado en la misma (Betanzos, Andrade \& Paz, 2006).

Así como las condiciones de trabajo en las que se encuentra inmerso el personal, el CO es uno de los elementos que interviene en el ambiente de trabajo en las organizaciones. Para esta investigación se contó con la participación de dos universidades públicas instaladas en el Estado de Guanajuato, aunque con orígenes y alcances distintos, teniendo como resultado diferencias en la cantidad de factores y reactivos que miden el CO en análisis exploratorios, sin embargo en análisis confirmatorios la estructura factorial del constructo se mantuvo. Cabe destacar que la estructura resultante fue validada para medir el constructo independientemente de la organización educativa que se trate, pues pese a que existen diferencias estadísticamente significativas en el factor afectivo entre las dos universidades, el resultado de ambos casos muestra que la confiabilidad se encuentra dentro de lo requerido. Sin embargo, considerando que las organizaciones pueden ser muy diferentes entre sí, será interesante poner a prueba este instrumento en organizaciones de otros sectores u otras regiones de México y América Latina. 


\section{Referencias}

Allen, N. \& Meyer, J. (1990). The measurement and antecedents of affective, continuance and normative commitment to the organization, Journal of Occupational Psychology, 63, 1-18. https://doi.org/10.1111/j.2044-8325.1990.tb00506.x

Allen, N. \& Meyer, J. (1996). Affective, Continuance, and Normative Commitment to the Organization: An Examination of Construct Validity, Journal of Vocational Behavior, 49, 252-276. https://doi.org/10.1006/jvbe.1996.0043

Arias, F. (enero-marzo 2001). El compromiso personal hacia la organización y la intención de permanencia: algunos factores para su incremento, Revista Contaduría y Administración, 200, 5-12.

Arbuckle, J. L. (2013). Amos (Versión 22.0) [Programa de cómputo]. Chicago: SPSS.

Barraza, A. \& Acosta, M. (octubre-diciembre 2008). Compromiso organizacional de los docentes de una institución de educación media superior, Innovación Educativa, 8(45), 20-35.

Betanzos, N., Andrade, P. \& Paz, F. (2006). Compromiso organizacional en una muestra de trabajadores mexicanos, Revista de Psicología del Trabajo y de las Organizaciones, 22(1), 25-43.

Blau, G. (2003). Testing for a four-dimensional structure of occupational commitment, Journal of Occupational and Organizational Psychology, 76, 469-488. https://doi.org/ 10.1348/096317903322591596.

Brown, T. (2006). Confirmatory Factor Analysis for Applied Research, EUA: The Guilford Press.

Cheung, G. W. \& Rensvold, R. B. (2002). Evaluating goodness-of-fit indexes for testing measurement invariance. Structural equation modeling, 9(2), 233-255.

Cohen, A. (1999). Relationships among five forms of commitment: an empirical assessment, Journal of Organizational Behavior, 20, 285-308. https://doi.org/10.1002/(SICI)1099-1379(199905) 20:3\&lt;285::AID-JOB887\&gt;3.0.CO;2-R 
Diedenhofen, B. \& Musch, J. (2016). COCRON: A web interface and $\mathrm{R}$ package for the statistical comparison of Cronbach's alpha coefficients. International Journal of Internet Science, 11, 51-60.

Davis, K. (1983). El comportamiento humano en el trabajo, México: McGraw Hill.

Figueroa, J., González, E. \& Solis, V. (1981). Una aproximación al problema del significado: las redes semánticas, Revista Latinoamericana de Psicología. 13 (3). 447-458.

Frutos de, B., Ruiz, M. \& San Martin, R. (1998). Análisis factorial confirmatorio de las dimensiones del compromiso con la organización, Psicológica, 19, 345-366.

Gómez-Sánchez, D., Recio, R. \& Avalos, M. (2012). Compromiso organizacional y su relación con la satisfacción laboral de los empleados en Rioverde, SLP. Retos y alcances de la Investigación Conductual 1er Congreso Nacional de Ciencias del Comportamiento.

Hinojosa, G. (2008). El tratamiento estadístico de las redes semánticas naturales, Revista Internacional de Ciencias Sociales y Humanidades, SOCIOTAM. XVIII(1), 133-154.

Lu, H., While, A. E. \& Barriball, K. L. (2005). Job satisfaction among nurses: A literature review. International Journal of Nursing Studies, 42, 211-227.

Mañas, M., Salvador, C., Boada, J., González, E. \& Agulló, E. (2007). La satisfacción y el bienestar psicológico como antecedentes del compromiso organizacional, Psicothema, 19(3), 395-400.

McElroy, J., Morrow, P. \& Laczniak, R. (2001). External organizational commitment, Human Resource Management Review, 11, 237256. https://doi.org/10.1016/S1053-4822(00)00050-4

Meyer, J. \& Allen, N. (1991). A three-component conceptualization of organizational commitment, Human Resource Management Review, 1(1), 61-89. https://doi.org/10.1016/1053-4822(91)90011-Z

Meyer, J. \& Herscovitch, L. (2001). Commitment in the workplace toward a general model, Human Resource Management Review, 11, 299-326. https://doi.org/10.1016/s1053-4822(00)00053-x 
Meyer, J., Stanley, D., Herscovitch, L. \& Topolnytsky, L. (2002). Affective, Continuance, and Normative Commitment to the Organization: A Meta-analysis of Antecedents, Correlates, and Consequences, Journal of Vocational Behavior, 61, 20-52. https:// doi.org/10.1006/jvbe.2001.1842

Morrow, P. (1983). Concept Redundancy in Organizational Research: The Case of Work Commitment, Academy of Management Review, 8(3), 486-500. https://doi.org/10.5465/amr.1983.4284606

Morrow, P. \& McElroy, J. (1986). Reseach notes on assessing measures of work commitment, Journal of Occupational Behaviour, 7 , 139-145. https://doi.org/10.1002/job.4030070206

Mowday, R., Steers, R. \& Porter, L. (1979). The Measurement of Organizational Commitment, Journal of Vocation Behavior, 14, 224-247. https://doi.org/10.1016/0001-8791(79)90072-1

Münch, L. \& García, J. (1990). Fundamentos de Administración. $5^{a}$ ed. México: Trillas.

Reyes, I. (1993), Las redes semánticas naturales, su conceptualización y su utilización en la construcción de instrumentos, Revista de Psicología Social y Personalidad, IX(1), 81-97.

Robbins, S. (1996). Comportamiento organizacional. México: Prentice Hall.

Vera, J., Pimentel, C. \& Batista, F. (2005). Redes semánticas: aspectos teóricos, técnicos, metodológicos y analíticos, Ra Ximbai, 1(003), 439-451.

Werther, W. \& Davis, K. (2000). Administración de personal y recursos humanos. $5^{a}$ ed. México: Mc Graw Hill

Recibido: 24 de febrero, 2017 Revisado: 28 de setiembre, 2017 Aceptado: 13 de octubre, 2017 